\title{
Short communication \\ On the irradiation creep by climb-enabled glide of dislocations
}

\author{
A.V. Barashev ${ }^{\mathrm{a}, \mathrm{b}^{*}}$, S.I. Golubov ${ }^{\mathrm{a}}$, R.E. Stoller ${ }^{\mathrm{a}}$ \\ ${ }^{a}$ Materials Science and Technology Division, ORNL, Oak Ridge, TN 37831-6138, USA \\ ${ }^{\mathrm{b}}$ Center for Materials Processing, Department of Materials Science and Engineering, University of \\ Tennessee, Knoxville, TN 37996-0750, USA
}

\begin{abstract}
In the climb-enabled glide model of irradiation creep, the plastic deformation is defined by the elastic deflections of pinned dislocations, which is an inconsistency. We argue that this relation is incorrect; instead, as in other pinning-unpinning-type models, the dislocations move from one set of obstacles to another, so that the inter-obstacle spacing determines creep rate, whereas the dependence on the applied stress is only implicit in the unpinning time.
\end{abstract}

Keywords: creep, metallic material, analytic functions, irradiation.

*Corresponding author. e-mail: abarashe@utk.edu (Alexander V. Barashev).

In a material with shear modulus $G$ under stress $\sigma$, the usual elastic strain $\varepsilon_{0}=\sigma / G$ is accompanied by the elastic strain $\varepsilon_{\mathrm{e}}=\varepsilon_{0} / 6$ due to the bowing-out network dislocations (see the work-hardening model by Mott [1] corrected by Friedel [2]). The unpinning of dislocations under irradiation results in continuous plastic deformation, i.e. creep. The Gittus climb-enabled glide model [3] of irradiation creep is one of the most frequently cited in the literature (see, e.g. in reviews [4,5]). It is one of the pinning-unpinning type models with two specific features: first, the unpinning mechanism is the dislocation climb, and, second, the plastic strain is associated with the dislocation elastic strain, $\varepsilon_{\mathrm{e}}$. The resulting creep rate is given by ${ }^{1}$

$$
\dot{\varepsilon}_{\text {Gittus }}=\varepsilon_{\mathrm{e}} / \tau=\sigma / 6 G \tau,
$$

where $\tau$ is the meantime for dislocation release from a pinning point.

In this communication, we argue that the association of the creep rate with the elastic strain is an implausible conjecture. According to Gittus [3] (p. 347, end of $2^{\text {nd }}$ paragraph): “...the nodes ... move forward permitting the line to straighten..." and next "When all points on the dislocations have climbed ... glide will have translated them all through a distance equal to the sagitta of a bowed dislocation". These statements overlook the fact that, when a node is released, the dislocation segment moves and produces larger strain, and after release of all pinning points, the dislocations glide to the next set of obstacles, where they bow out again. Obviously, the area swept by a bowed dislocation is translated into the plastic component of the strain; but there must be an additional plastic contribution to strain when the dislocation straightens, which is not accounted for in such an approach. Below, we consider the deformation process to clarify this issue.

Consider plastic deformation of a crystal with a dislocation network of size $L=(\rho / 3)^{-1 / 2}$ of dislocations of density $\rho$ and the Burgers vector $b$ (see Fig. 1). Under an applied shear stress, the locked dislocation segments bow out until the force due to stress is balanced by the line tension force, sweeping an area $S_{0}=\sigma L^{3} / 6 G b$ (see details in $[1,2]$ ) per a network cell, which corresponds to the elastic strain $\varepsilon_{\mathrm{e}}$. Consider dislocation segments in two adjacent network cells (solid blue line BD in Fig. 1). The initial strain $\varepsilon_{\mathrm{e}}$ in these cells is formed by the total area swept by dislocation $2 S_{0}$ (per volume $2 L^{3}$ ). Assume that the middle pinning point (C) is released. Then the new chord of arc (dashed blue line BD) of the

\footnotetext{
${ }^{1}$ In the Mott's [1] paper $\varepsilon_{\mathrm{e}}=\varepsilon_{0} / 24$ rather than true $\varepsilon_{0} / 6$, due to a numerical flaw corrected by Friedel in [2]. Gittus [3] misinterpreted Mott's definition for the density of dislocation segments, $N$, by using three times higher value, which resulted in $\varepsilon_{\mathrm{e}}=\varepsilon_{0} / 2$. Heald and Harbottle [6] omitted the remaining factor $1 / 2$ and used $\varepsilon_{\mathrm{e}}=\varepsilon_{0}$ (this was reproduced in the review by Matthews and Finnis [4]). Here, we use the correct value $\varepsilon_{\mathrm{e}}=\varepsilon_{0} / 6$.
} 
combined dislocation segment is double in length, $L^{\prime}=2 L$, hence the area swept by dislocation, $\propto\left(L^{\prime}\right)^{3}$, increases to $8 S_{0}$, corresponding to strain $4 \varepsilon_{\mathrm{e}}$. (The Gittus model assumes EE' which is wrong in our view.) The strain increase is, thus, $3 \varepsilon_{\mathrm{e}}$, which exceeds the value $\varepsilon_{\mathrm{e}}$ predicted by Gittus for the end of the dislocation unpinning process. Release of more obstacles will result in even greater deviation from the Gittus prediction. Moreover, for a simple case drawn in Fig. 1, the strain remains reversible (i.e. elastic) until all the pinning points released and the dislocation moved to the next row of obstacles (A'-D' in Fig. 1). The latter is the true equivalent state, when again the dislocations produce the elastic strain $\varepsilon_{\mathrm{e}}$, the same as before unpinning, and which does not contribute to the plastic deformation. The gliding distance between these two states, $L$, corresponds to the plastic strain $\varepsilon_{\mathrm{p}}=b L^{2} / L^{3}=b(\rho / 3)^{-1 / 2}$, hence the strain rate is

$$
\dot{\varepsilon}=\varepsilon_{\mathrm{p}} / \tau=b \sqrt{\rho / 3} / \tau .
$$

The same result is obtained from the general equation for stain rate

$$
\dot{\varepsilon}=\rho_{\mathrm{m}} b V,
$$

where $\rho_{\mathrm{m}}$ is the mobile dislocation density and $V$ is the dislocation velocity ( $=L / \tau, L$ is the inter-obstacle distance), with $\rho_{\mathrm{m}}=\rho / 3$ and $L=(\rho / 3)^{-1 / 2}$ in the case considered.

Eq. (2) gives much higher strains than the Gittus model, e.g. an order of magnitude higher: $\varepsilon_{\mathrm{p}}=10 \varepsilon_{\mathrm{e}}$, for typical values of $b=2 \times 10^{-10} \mathrm{~m}, \rho=10^{14} \mathrm{~m}^{-2}, \sigma=100 \mathrm{MPa}$ and $G=80 \mathrm{GPa}$. Importantly, Eq. (2) has no explicit dependence on the applied stress. The latter well-documented dependence (see, e.g. in [7]) must be present implicitly in the unpinning time, as, e.g. in the model by Kelly and Foreman [8].

\section{Acknowledgements}

The research was sponsored by a research grant from the Office of Fusion Energy Sciences U.S. Department of Energy, under contract DE-C05-00OR22725 with UT-Battelle, LLC.

\section{References}

[1] N.F. Mott, Phil. Mag. 43 (1952) 1151-1178.

[2] J. Friedel, Phil. Mag. 44 (1953) 444-448.

[3] J.H. Gittus, Phil. Mag. 25 (1972) 345-354.

[4] J.R. Matthews, M.W. Finnis, J. Nucl. Mater. 159 (1988) 257-285.

[5] R.A. Holt, J. Nucl. Mater. 372 (2008) 182-214.

[6] P.T. Heald, J.E. Harbottle, J. Nucl. Mater. 67 (1977) 229-233.

[7] E.R. Gilbert, J.F. Bates, J. Nucl. Mater. 65 (1977) 204-209.

[8] B.T. Kelly, A.J.E. Foreman, Carbon 12 (1974) 151-158.

\section{Figure caption}

Fig. 1. A network of dislocations of density $\rho$ under stress. The dislocations form arcs of radius $r$ (solid blue lines A-D). When the pinning point $C$ is released, an arc of the same radius is formed (dashed blue line B-D). When all the obstacles are released, the dislocation glides to the next row of obstacles (A'-D'). 


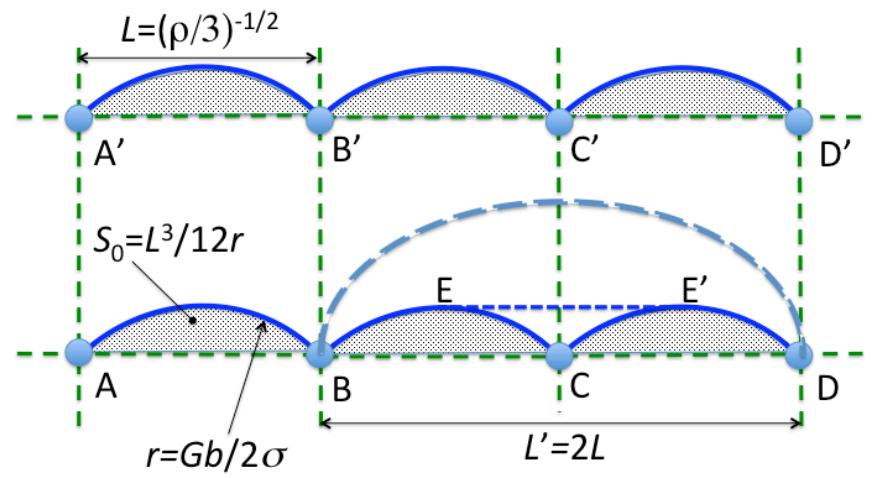

